\title{
New horizons for mental health research in intellectual disabilities
}

We are honoured to follow in the footsteps of Sally-Ann (Anna) Cooper, who, after 10 years, gave up the role of Editor of the Mental Health editions of JIDR at the end of 2016. Anna has made a significant contribution to the quality and extent of the research into mental disorders in individuals with intellectual disabilities published in the journal, and ensured an important focus on the clinical aspects, diagnosis, and epidemiology of mental disorders in intellectual disabilities. We are keen to continue the trajectory established by Anna Cooper and her predecessors, and believe that the ongoing efforts to understand the underlying causes of intellectual disabilities and associated mental disorders will have an increasing impact upon the management of these comorbidities, and help to improve the quality of life of individuals with intellectual disabilities.

One major barrier to improved management of mental disorders in individuals with intellectual disabilities is the substantial lack of evidence for effective treatments. While not all treatments require randomised-trial level evidence if it has already been shown to be efficacious in other populations, it is important to demonstrate efficacy if the treatment is unique, has required substantial adaptation, or if there are reasons to believe that efficacy and the risk/ benefit ratio may differ when used in individuals with intellectual disabilities. This issue of the Journal of Intellectual Disabilities Research includes two reports of randomised trials. It is encouraging that such trials are now much more commonly undertaken, thus heralding a new horizon for the treatment of mental health issues in individuals with intellectual disabilities by expanding the evidence-base, particularly with regards to the management of challenging behaviour.

One of these trials was a cluster randomised trial of the effectiveness of nidotherapy (an intervention aiming to address environmental factors that may be related to aggressive challenging behaviour) [ref - JIR 12360], while the other examined the clinical efficacy of a combination of multisensory environment and massage therapy for challenging behaviour in adults with severe intellectual disability [JIR12377]. Both these trials showed some evidence of efficacy for these interventions. However, the nidotherapy intervention took 8 months to have an effect, which the authors attributed to it being delivered by staff. It may therefore be reasonable to assume that the intervention could have taken a while to be implemented. Nevertheless, the long delay in effect raises questions about the mechanism of action, and might also be a chance finding. Further research may be required to target the environmental factors involved in the initiation and maintenance of aggressive behaviour specifically for a more rapid response, before attempting a replication of the trial.

The second trial was a pilot randomised controlled trial with four arms, and thus not powered to demonstrate efficacy [JIR12377]. Indeed, there was no effect for any of the interventions (multisensory environment, massage therapy or its combination) on the primary outcome measures of challenging behaviour in individuals with severe intellectual disabilities after 10 weeks of intervention, but the authors reported that ratings on a secondary measure of "alertness" showed that individuals in two of the treatment arms (multisensory environment, or combination intervention) experienced a significant change from active to inactive states during the interventions. This was interpreted as evidence for a state of "passive alertness", assuming that activity levels and alertness are surrogate markers of agitation and thus challenging behaviour. The 
positive effect was short term and only demonstrated during the intervention rather than after a delay, and was not measured blind to the intervention. Nevertheless, a larger trial with careful consideration of outcome measures, blinding and sample size may be warranted.

Clinical trials in intellectual disabilities are often complex and difficult to conduct, and the results may be affected by the relative scarcity of valid and reliable outcome measures. We are keen to support the publication of articles reporting on the development and validation of reliable outcome measures for trials and practice, such as the psychological therapies outcome scale - ID (PTOS-ID) reported in this issue [JIR12361]. The authors recommend further work to establish its reliability and ability to measure change.

Biomarkers can also be used to show whether a new or adapted treatment acts through/ upon an assumed mechanism, thus providing proof-of-principle before embarking on full-scale trials. Such approaches are rarely used in ID research, but their usefulness is demonstrated by a study of slowpaced breathing, a simple but potentially effective stress-management strategy that often form part of interventions for stress and anxiety [JIR12350]. These authors measured cardiac efferent vagal tone (through heart rate variability) to show that this technique can potentially reduce stress in young individuals with intellectual disabilities.

A better understanding of the mechanisms of mental disorders in ID might be gleaned by in-depth study of the phenotypes associated with different intellectual disability genetic syndromes, to identify causative genes as well as common pathways. This issue includes a comprehensive review of the clinical and genetic aspects of 15q11.2 BP1-2 microdeletion syndrome, an emerging condition with over 200 individuals reported in the literature [JIR12382]. The psychiatric disturbances commonly reported in individuals with 15q11.2 BP1-2 microdeletion syndrome include Autism spectrum disorders, psychosis or schizophrenia, OCD and ADHD. The BP1-2 locus form part of the Prader-Willi/ Angelman syndrome region on chromosome 15, but contains only 4 genes, including the CYFIP1 gene whose product interacts with FMRP, the protein associated with fragile $X$ syndrome, demonstrating how the same biological pathway may be involved in different genetic syndromes.

We will continue to support the publication of high quality clinical, epidemiological and health services research that helps to improve the recognition and definition of mental disorders in intellectual disabilities, such as the report highlighting the nature and prevalence of catatonic symptoms in young people with autism, which may be more common than previously thought [JIR12362]. In the next few years we would like to see more articles that provide an understanding of the mechanisms underlying mental disorders, and those reporting a more in-depth understanding of the phenotypes that can help to target treatments more effectively. We believe that the time has come for conducting randomised trials of potential treatments to improve the existing evidence base, and we look forward to publishing more such reports. 